\title{
Boundary element methods in the prediction of the acoustic damping of ship whipping vibrations
}

\author{
D. S. Holloway G. A. Thomas M. R. Davis*
}

(Received 8 August 2003)

\begin{abstract}
Damping of ship whipping vibrations following a slam due to wave impact is traditionally assumed to be primarily of material or structural origin. However, several mechanisms of energy dissipation to the surrounding water exist, including gravity and acoustic waves. Neither transports much energy for the lowest frequency modes, in which the acoustic wavelength may be an order or magnitude greater than the ship length whereas the gravity wavelength is at least an order of magnitude shorter than the ship beam. However, the acoustic damping ratio increases as the fourth power of frequency, becoming significant for higher frequency modes. This paper investigates at what
\end{abstract}

*School of Engineering, University of Tasmania, Hobart, Australia. mailto: Damien. Holloway@utas. edu .au

See http://anziamj.austms.org.au/V45/CTAC2003/Hol2/home.html for this article, (C) Austral. Mathematical Soc. 2004. Published August 10, 2004, amended August 11, 2004. ISSN 1446-8735 
frequencies acoustic damping is significant for a typical ship. The acoustic problem is solved using a three-dimensional exterior boundary element method, which is described. Acoustic damping is then compared with total damping from full scale ship measurements and material damping from laboratory tests, from which the relative contributions are deduced.

\section{Contents}

1 Introduction

C846

2 The acoustic problem

C848

3 The flexural problem

C850

4 Test case - equivalent ship

C851

5 Results and conclusions

C852

References

C856

\section{Introduction}

Global strain records on ships clearly indicate there are two significant loading regimes [6]. Predominantly one observes an underlying response to the encountered waves at the frequencies of significant wave excitation forces. These frequencies are generally much lower than the ship's flexural natural frequencies, therefore the response may be regarded as quasi-static for structural analyses, including only dynamic effects due to the ship's rigid body motions. The other major loading pattern observed is the transient hull whipping response (global flexural vibration) to impulsive excitations 
from "slams", severe short duration impacts, typically near the bow, resulting from the coincidence of unusually large wave crests and plunging bow motions. These may be particularly severe for impacts with the cross deck structure of catamarans, and the severity and frequency of these events is increased for high speed vessels.

The structural response of a ship to slams is critical to design. A severe slam is likely to represent the ultimate load experienced by a ship, and single events have been known to cause permanent global buckling of the ship hull plating and frames [5]. However, of at least equal concern is the fatigue life reduction due to the accumulated effects of moderate slams. To this end it is important to know the damping characteristics of the whipping vibrations.

Damping arises from many sources, originating in both the structure and surrounding fluid. The former includes the inherent hysteresis of the hull material, and substantial additional damping arising from the built up structure (for example, effects of joints and welds, cladding, fittings, engine and superstructure mounts, and cargo). Fluid damping sources include free surface gravity wave generation (which is significant only at the relatively lower frequencies of the rigid body motions), acoustic wave radiation (the subject of this paper) and viscous effects (small). Whereas acoustic damping is negligible at low frequencies, it will be shown that it becomes significant for higher frequency modes, to the extent that these modes may be ignored in a hydroelastic analysis.

The current research arises from collaborative work with Incat, a manufacturer and exporter of state of the art large high speed catamaran passenger ferries. Recent ships built by Incat approached $100 \mathrm{~m}$ in length, with service speeds of up to $45 \mathrm{kt}$. The results presented in this paper will therefore focus on ships of this type. However, the modal analysis is simplified by the use of an equivalent prismatic ship. 


\section{The acoustic problem}

Propagation of small amplitude acoustic waves through water at constant frequency is governed by the Helmholz equation, a condition of compatibility of velocity on the surface of the radiating source (ship hull), zero pressure on the free surface (due to the great disparity between the densities and speeds of sound in air and water) [4], and a far-field condition of outgoing waves,

$$
\begin{aligned}
\nabla^{2} \phi+k^{2} \phi & =0 \quad \text { in the fluid domain, } \\
\nabla \phi \cdot \mathbf{n} & =\mathbf{V} \cdot \mathbf{n} \quad \text { on the hull surface, } \\
\phi & =0 \quad \text { on } z=0 \\
\lim _{r \rightarrow \infty} r\left(\frac{\partial \phi}{\partial r}-\mathrm{i} k \phi\right) & =0
\end{aligned}
$$

where $k=\omega / c$ is the wavenumber and $\omega$ and $c$ are respectively the angular frequency and wave speed. For harmonic waves $\phi=\Re\left(\phi_{0} e^{-\mathrm{i} \omega t}\right)$, pressure is $p=-\rho \partial \phi / \partial t$, and local velocity $\mathbf{v}=\nabla \phi$.

The water-air interface is acoustically flat as the predominant gravity wavelengths are very short compared with the acoustic wavelengths, enabling a double body representation of the ship hull in which corresponding points on the body and image have identical velocity, automatically satisfying (3).

We use a boundary element method based on Kirkup's [2]. The method uses the point source function

$$
G_{k}(r)=\frac{1}{4 \pi} \frac{e^{\mathrm{i} k r}}{r}
$$

which satisfies (1) and (4). The body surface is discretised into triangular elements over which sources of this form are distributed piecewise uniformly with time-complex intensities $\left\{\phi_{0}\right\}$. The induced velocity at any point is determined by integration of the gradient of (5) over each element using 
Gaussian quadrature, hence (2) is satisfied at the centroid of each element by determining $\left\{\phi_{0}\right\}$ in

$$
\left[M-\frac{1}{2} I\right]\left\{\phi_{0}\right\}=[L]\left\{\mathbf{V} \cdot \mathbf{n}_{i}\right\}
$$

where

$$
\begin{aligned}
L_{i j} & =\int_{\text {element } j} G_{k}\left(r_{i j}\right) d S, \\
M_{i j} & =\int_{\text {element } j} \nabla G_{k}\left(r_{i j}\right) \cdot \mathbf{n}_{j} d S,
\end{aligned}
$$

$\mathbf{n}_{i}$ and $\mathbf{n}_{j}$ are the normals to elements $i$ and $j$ respectively, and $r_{i j}$ is the distance between the centroid of element $i$ and a point on element $j$.

Given $\left\{\phi_{0}\right\}$, hence surface pressure $p=-\mathrm{i} \rho \omega \phi_{0}$, the average radiated power

$$
P=\Re \int \frac{1}{4} \bar{p} \mathbf{V} \cdot \mathbf{n} d S=\sum_{\text {all elements }} \frac{1}{4} \rho \omega A \times \Im\left(\bar{\phi}_{0} \mathbf{V} \cdot \mathbf{n}\right),
$$

$\left(A=\right.$ element area). This includes a factor $\frac{1}{2}$ to reduce the double body representation to the actual submerged hull. Finally damping is obtained from the energy radiated per cycle, $2 \pi P / \omega$.

The linear equations (6) become singular at frequencies corresponding to the eigenvalues of $[2 M]$, and ill-conditioned in the vicinity of these frequencies. These are the resonant frequencies of the corresponding interior acoustic problem. Kirkup [2] therefore uses a hybrid method using a linear combination of (6) and its derivative with respect to $\mathbf{n}_{i}$. This is unnecessary for the present problem due to the long wavelength to ship length ratios involved, and was found by validation against the analytic solution for a oscillating sphere at low frequency [3] to be detrimental to the accuracy of the solution at such low frequencies.

The speed of sound in water adopted for the present calculations was $1447 \mathrm{~m} / \mathrm{s}$, corresponding to fresh water of density $1000 \mathrm{~kg} / \mathrm{m}^{3}$ at $10^{\circ} \mathrm{C}$. 


\section{The flexural problem}

Structural frequencies and mode shapes are required for $\mathbf{V}$ in (6), and in turn the fluid surrounding the hull affects the structural response. However, hydrodynamic effects are separated into damping, which has a negligible influence on the modal analysis, and added mass effects, which we will assume may be incorporated into an effective structural mass, varying (in accordance with slender body theory) primarily with the local hull cross section. Thus the flexural problem is solved independently of the acoustic one.

The equation of motion for a straight slender beam of length $L$, mass distribution $\mu(x)$ (including added mass), bending stiffness $E I(x)$, and negligible damping, obtained by applying dynamic equilibrium to an element of length $d x$ and using the moment-curvature relation for a beam, is

$$
\mu \frac{\partial^{2} u(x, t)}{\partial t^{2}}=-E I \frac{\partial^{4} u}{\partial x^{4}}
$$

which for a uniform beam (constant $\mu$ and $E I$ ) and with initial conditions $u(x, 0)=0$ has solutions of the form

$$
u(x, t)=\sin \omega t\left(A e^{k x}+B e^{-k x}+C \cos k x+D \sin k x\right),
$$

where $\omega=k^{2} \sqrt{E I / \mu}$. Applying free end boundary conditions $\left(\partial^{3} u / \partial x^{3}=\right.$ $\partial^{2} u / \partial x^{2}=0$ at $x=0, L$ and all $t$ ) leads to

$$
\left[\begin{array}{cccc}
1 & 1 & -1 & 0 \\
1 & -1 & 0 & -1 \\
e^{\theta} & e^{-\theta} & -\cos \theta & -\sin \theta \\
e^{\theta} & -e^{-\theta} & \sin \theta & -\cos \theta
\end{array}\right]\left[\begin{array}{l}
A \\
B \\
C \\
D
\end{array}\right]=\left[\begin{array}{l}
0 \\
0 \\
0 \\
0
\end{array}\right],
$$

where $\theta=k L$. Setting the determinant of the above matrix equal to zero, and after some reduction, we obtain the implicit expression $\cos \theta=1 / \cosh \theta$, giving the first six frequencies in the ratio $1: 2.76: 5.40: 8.93: 13.34: 18.64$. 
TABLE 1: Hull dimensions

\begin{tabular}{|l|cc|}
\hline & $\begin{array}{c}\text { Incat 86m } \\
\text { wavepiercer }\end{array}$ & $\begin{array}{c}\text { Equivalent } \\
\text { rectangular ship }\end{array}$ \\
\hline Length $(\mathrm{m})$ & 86.4 & 80 \\
Demi-hull beam $(\mathrm{m})$ & 4.5 & 4 \\
Draught $(\mathrm{m})$ & 2.704 (excluding keel) & 1.6 \\
Displacement $\left(\mathrm{m}^{3}\right)$ & 1030 & 1024 \\
Hull offset from & & \\
vessel centreline $(\mathrm{m})$ & 10.8 & 10 \\
\hline
\end{tabular}

Finally, for each frequency parameter $\theta$,

$$
\begin{aligned}
A & =\frac{-\sin \theta+\cos \theta-e^{-\theta}}{4(\sinh \theta-\sin \theta)}, \\
B & =\frac{1}{2}-A, \\
C & =\frac{1}{2}, \\
D & =2 A-\frac{1}{2},
\end{aligned}
$$

determines the mode shapes normalised by $u(0, t)$.

Modal energies, required for calculation of damping ratios, can be shown for normalised amplitudes to be

$$
E=\int_{0}^{L} \frac{1}{2} \mu\left(\frac{\partial u(x, 0)}{\partial t}\right)^{2} d x=\frac{1}{8} \mu L \omega^{2} .
$$

\section{Test case - equivalent ship}

The particular ship of interest is an Incat $86 \mathrm{~m}$ wavepiercing catamaran. However, as the main purpose of this study was to investigate at what frequencies 
TABle 2: Contributions to damping of first longitudinal mode, Incat vessel

\begin{tabular}{|l|c|}
\hline Damping source & \% of total \\
\hline Acoustic waves & 0.07 \\
Gravity waves & 0.7 \\
Water viscosity & 0.2 \\
Material (Aluminium) & 5 \\
Structure & 94 \\
\hline
\end{tabular}

acoustic damping may be significant, a nominally equivalent catamaran with rectangular hulls was modelled to simplify the modal analysis. This is unlikely to significantly affect the conclusions as deviation from the actual ship would be at a length scale substantially shorter than the acoustic wavelength. Table 1 gives hull details.

The first natural frequency of the equivalent ship $(3 \mathrm{~Hz})$ was nominally matched to that of the actual ship by appropriate choice of $E I$, and added mass was assumed to be equal to the displaced ship mass (which only affects the modal energies slightly, but nevertheless is a realistic assumption). In addition, the stiffness was varied to investigate the effect of natural frequencies higher or lower by a factor of 2 .

\section{$5 \quad$ Results and conclusions}

Figure 1 shows the dramatic increase of damping ratio $(\zeta=P / 2 \omega E)$ with $\omega$, averaging a rate of $\omega^{4}$. This compares with the analytic result of Lamb [3] for a vibrating arbitrarily rigid solid at low frequency, which leads to $\zeta$ increasing with $\omega^{3}$. The difference arises from the body's flexure. The flexural wavelength is much shorter then the acoustic wavelength, so that the near-body fluid motion is one of alternate sloshing between adjacent flexural anti-nodes, producing relatively little net motion in the far field, hence little 


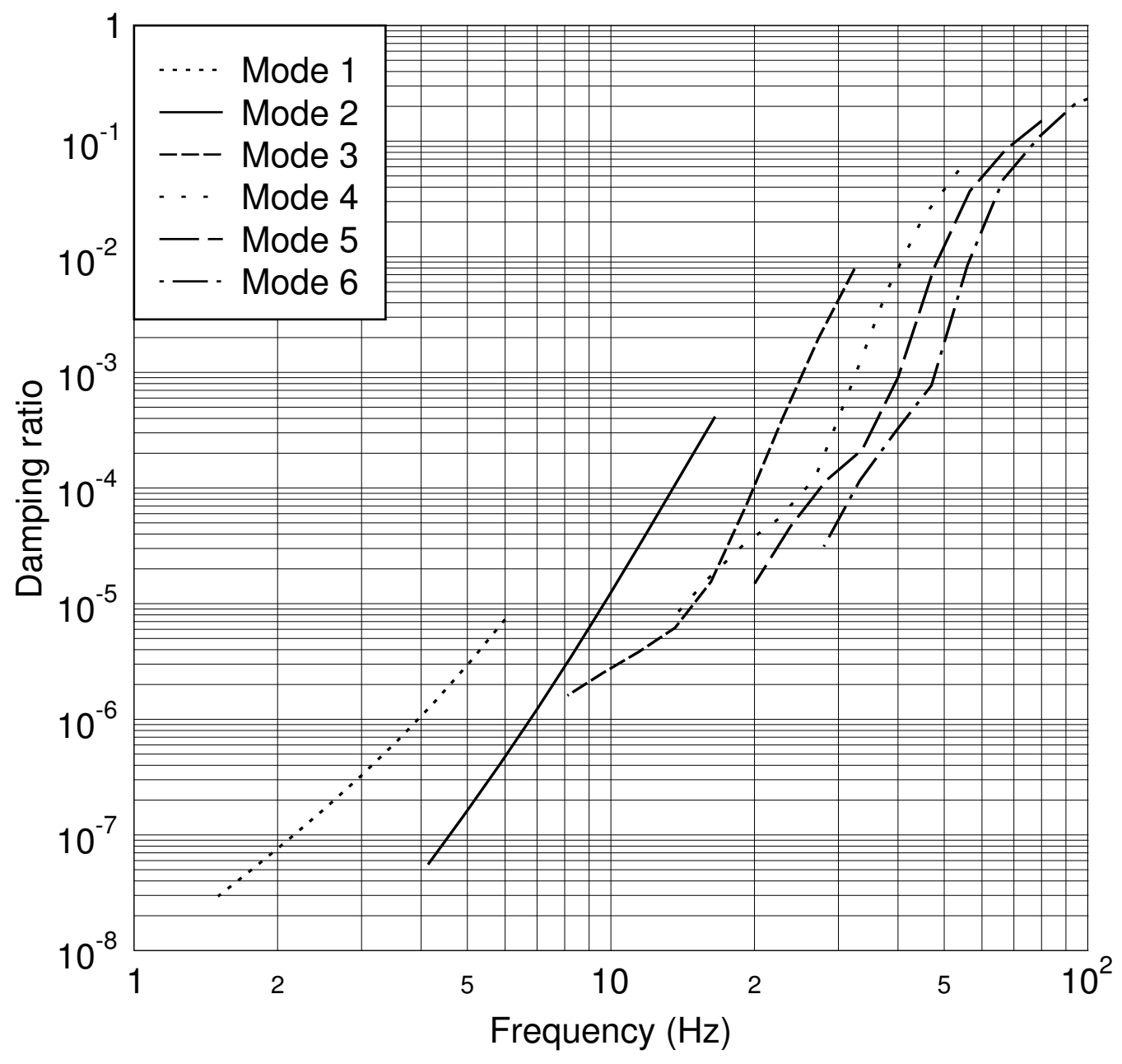

FiguRE 1: Damping ratio as function of frequency and mode number. 


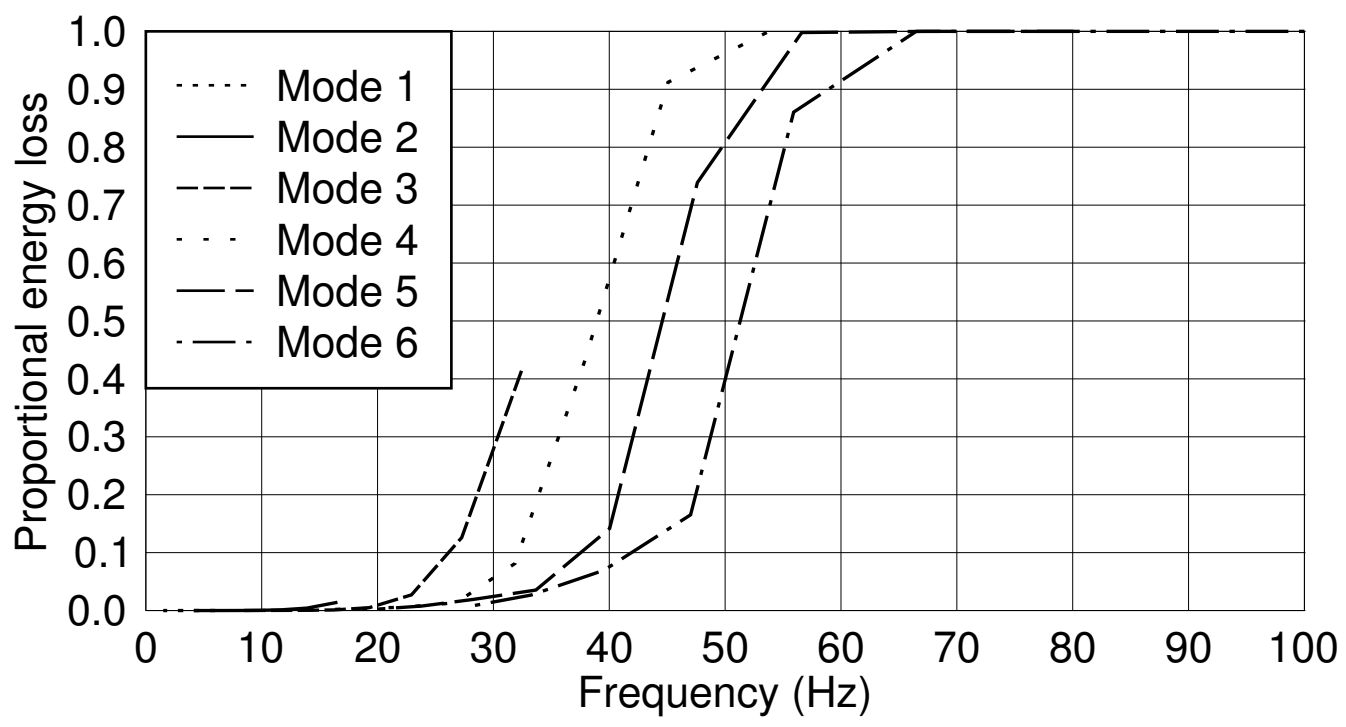

Figure 2: Proportion of modal energy lost during one oscillation cycle of the first mode. 
radiated power. This disparity between flexural and acoustic wavelengths increases at lower frequencies, causing a stronger dependence of $\zeta$ on $\omega$ than for the rigid body case. This explanation is also consistent with the drop in $\zeta$ with mode number at fixed frequency.

The damping ratios of Figure 1 are used to compute the proportion of modal energy that would be lost during a single cycle of the first mode, (about $0.33 \mathrm{~s}$, comparable to the duration of a typical slam impact), and these are shown in Figure 2. It is evident from this figure that the rate of decay of the 6th mode at its nominal frequency of $56 \mathrm{~Hz}$ is so rapid as to make that mode (and higher ones) insignificant in the impulse response of the ship.

Table 2 compares acoustic damping with other sources. In this comparison, total damping and material damping were measured respectively from full scale anchor drop tests and simple beam vibration tests, viscous and gravity wave calculations used methods described respectively in [7] and [1], and structural damping was assumed to be the remainder. However, if other forms of damping do not change with frequency, then based on the computed increase with mode number shown in Figure 1 acoustic radiation would account for about $20 \%$ of total damping for mode 4 , and $95 \%$ of total damping for mode 6 .

\section{Summary:}

- acoustic damping is negligible for the first mode compared with other sources of damping, predominantly structural;

- acoustic damping becomes significant at about $10 \times$ the first mode frequency;

- the damping ratio increases approximately with $\omega^{4}$; 
- damping is high by the sixth longitudinal mode. Higher modes can therefore be ignored in the hydroelastic analysis of ships similar to the one in this study.

\section{References}

[1] D. S. Holloway and M. R. Davis. Green function solutions for the transient motion of water sections. Journal of Ship Research, 46(2):99-120, 2002. C855

[2] Stephen Kirkup. The Boundary Element Method in Acoustics. Integrated Sound Software, 1998. C848, C849

[3] Sir Horace Lamb. The Dynamical Theory of Sound. Dover, 1960. Republication of 2nd (1925) edition. C849, C852

[4] Allan D. Pierce. Acoustics. McGraw-Hill, 1981. C848

[5] Giles Thomas, Michael Davis, Damien Holloway, and Tim Roberts. Transient dynamic slam response of large high speed catamarans. In 7th International Conference on Fast Sea Transpoartation, volume 2, pages B1:1-8, 2003. C847

[6] Giles Thomas, Michael Davis, Damien Holloway, and Tim Roberts. The whipping vibration of large high speed catamarans. International Journal of Maritime Engineering, 145(A4), 2003. C846

[7] Giles A. Thomas. The Slamming of Large High Speed Catamarans. PhD thesis, University of Tasmania, 2003. C855 